\title{
Paper
}

\section{Study of Visibility in a Simulated Fog Environment}

\author{
Fanghui XU*,**, Zijie WANG*,**, Zheqian ZHANG*,**, Xiaoli ZHOU*,**,† and Muqing LIU*,**
}

* Department of Light Source and Illuminating Engineering, Fudan University, 220 Handan Rd, Shanghai 200433, People's Republic China

** Engineering Research Center of Advanced Lighting Technology, Ministry of Education, Shanghai 200433, People's Republic China

Received December 1, 2016, Accepted March 1, 2017

\begin{abstract}
Actual illumination values calculated in bad weathers may be significantly lower than theoretically calculated values. Therefore, it is necessary to investigate visibility under hazy conditions. In this study, a simulated fog environment is established for this purpose. It was observed that human visual acuity is strongly correlated with the level of fog density. The results obtained may contribute to the design of street lighting.
\end{abstract}

KEYWORDS: fog, visibility, transmittance, astigmatism, visual acuity

\section{Introduction}

Studies on target visibility based on lighting streets or other environments are usually performed under ideal conditions, i.e., the gaseous medium (usually air) is dry and clean and attenuation of light energy is neglected. However, the current levels of fog and haze (regarded as atmospheric aerosols) appear to have increased, decreasing the actual illuminance. Thus, several researchers have studied the transmittance properties of light under such conditions ${ }^{1-8)}$, wherein variables, including wavelength, color temperature, and light source, were considered. The results show that incandescent lamps exhibit good transmittance in fog conditions, and yellow LEDs offer the best performance among all LED colors. However, the effect of fog and haze on human eyesight is seldom mentioned. In this study, visibility in simulated fog environments of different densities is evaluated. The relationship between visual acuity and fog density is revealed. Some characteristics of the human eye are found to influence visual acuity under different fog densities. The results may contribute to the design of street lighting or illumination in other situations involving fog and haze.

\section{Experimental setup}

\subsection{Experimental devices}

To evaluate visibility, a uniform, stable, and controllable fog environment was established. Figure 1 illustrates the schematic of the experimental system. Except humidifiers, other devices were placed inside a large opaque cardboard box. The box, which was $3.0 \mathrm{~m}$ in length, $0.7 \mathrm{~m}$ in height, and $1.2 \mathrm{~m}$ in width, was made of wooden planks, wooden frames, and black plastic boards. Two windows comprising acrylic plates were

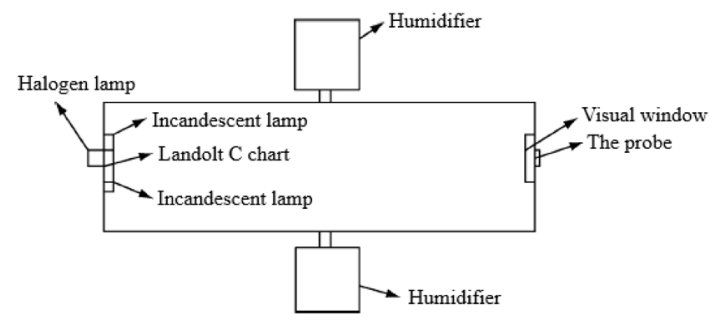

Figure 1 Schematic of the experimental system.

mounted at the ends of the dark box. The interior of the box was painted black except for the acrylic windows to prevent any reflection of light.

Devices have different functions. A halogen lamp was used as the reference light source to determine the level of fog density, as outlined in the literature ${ }^{1-8)}$. Since the percentage of fog density, $P$, was closely related to the transmittance of the light source, $T$, the fog density was calculated using Eq. (1):

$$
P=1-T \text {. }
$$

The transmittance of the light source is defined by Eq. (2), where $E^{\prime}$ denotes the illuminance in the simulated fog environment and $E_{0}$ is the illuminance in the absence of fog:

$$
T=E^{\prime} / E_{0} \text {. }
$$

The Landolt $\mathrm{C}$ chart, which is commonly used to examine visual acuity, was used as the observation target. Two desk lamps (Philips E27, 40-W incandescent lamps with a color temperature of $2,666 \mathrm{~K}$ ), installed symmetrically at the same height, were used to obtain uniform and stable illumination of the target.

Two ultrasonic humidifiers were utilized to produce

${ }^{\dagger}$ Corresponding Author: Xiaoli Zhourzhouxl@fudan.edu.cn 
a uniform, stable, and controllable fog environment. Minute droplets of water were formed by leaving the atomization component of the humidifiers in highfrequent resonance with an ultrasonic wave $(1.7 \mathrm{MHz})$ in order to simulate a fog environment. The average diameter of the water droplets is about $5 \mu \mathrm{m}$, which is in agreement with the water-droplet diameter of fog in nature (between $4 \mu \mathrm{m}$ and $10 \mu \mathrm{m}$ ).

Light produced by the lamps at the left end of the box passed through the simulated fog environment and reached the right end of the box. The probe was used to detect the illuminance value, and the percentage of fog density was calculated using the equations mentioned above. Subjects read the Landolt $\mathrm{C}$ chart through the visual window. The visual acuity of all subjects should be above 0.8 out of 1.5 .

\subsection{Experimental procedure}

1. Experimental preparations: The lamps (halogen lamp and incandescent lamps) were preheated until their illuminance values were stable. Subjects wore eye patches and were seated such that they could see through the visual window; they were allowed to adapt to the dark environment for about 2 min.

2. Recording the initial values: The desk lamps were switched off and the initial illuminance, $E_{0}$, produced only by the halogen lamp was recorded. Furthermore, the halogen lamp was switched off and the desk lamps were switched on. Subjects were asked to observe the Landolt $\mathrm{C}$ chart through the visual window and inform the operator which lines they can read. Their initial visual acuity was recorded.

3. Simulating a fog environment: The desk lamps were switched off and the halogen lamp was switched on. The two humidifiers were switched on. When the illuminance was about 5 $\mathrm{lx}$ (the initial illuminance of the halogen lamp is normally between 78 and $80 \mathrm{~lx}$ ), the humidifiers were switched off. During the humidifying process, the subjects were asked to adapt to the dark environment as they did in preparation.

4. Recording the illuminance: After the illuminance reached $51 x$, the halogen lamp was switched off and the desk lamps were switched on. The subjects

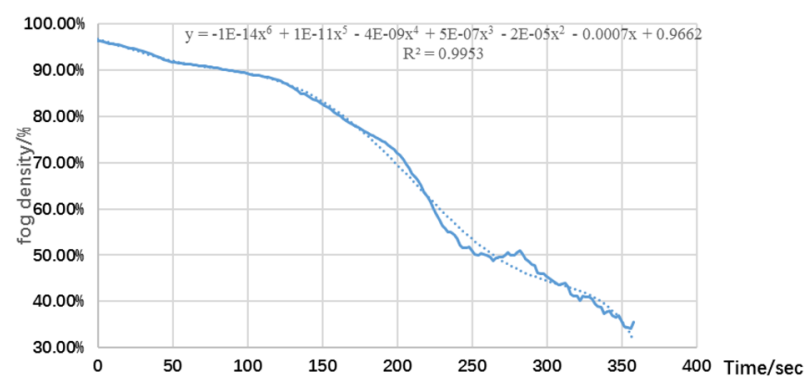

Figure 2 Relationship between time and fog density. were allowed to observe the chart. The simulated fog slowly dissipated as time lapsed. When the subjects were able to identify the 0.3 line clearly, they were prompted to close their eyes. Then, the desk lamps were switched off and the halogen lamp was switched on. The operator recorded the illuminance as quickly as possible. Illuminance for different visual acuity values $(0.4,0.5,0.6$, and 0.8$)$ was recorded until the subjects restored their sight.

5. After recording all illuminance values, all the lamps were switched off. Noting that only one subject was tested at a time, subsequent experiments were not performed until the fog vanished completely and the glass of the visual window was cleaned.

Before the experiment, the change in the density of fog with elapsed time was measured, as shown in Figure 2.

\section{Results and discussion}

In this study, 32 subjects aged 20-30 years, including 20 males and 12 females (among them, 17 having an initial visual acuity below 1.0 and 8 having an initial visual acuity above 1.0), were tested. All the initial visual acuity tested is the corrected vision. Table 1 lists the fog densities corresponding to various visual acuities of subjects $(0.3,0.4,0.5,0.6$, and 0.8$)$. The standard deviation of fog density ranges from 8 to $20 \%$, which is high. One possible reason is that there are significant differences between the observations of the subjects. Moreover, visual acuity increases as fog density decreases.

From Table 1, it can be noted that visual acuity

Table 1 Relationship between fog density and visual acuity.

\begin{tabular}{lrcccc}
\hline Subject's visual acuity & 0.3 & 0.4 & 0.5 & 0.6 & 0.8 \\
The average value of fog density & $77 \%$ & $70 \%$ & $60 \%$ & $46 \%$ & $34 \%$ \\
The standard deviation of fog density $\sigma$ & $8 \%$ & $11 \%$ & $16 \%$ & $20 \%$ & $18 \%$ \\
The maximum value of fog density & $85 \%$ & $84 \%$ & $84 \%$ & $78 \%$ & $72 \%$ \\
The minimum value of fog density & $63 \%$ & $47 \%$ & $31 \%$ & $16 \%$ & $12 \%$ \\
\hline
\end{tabular}

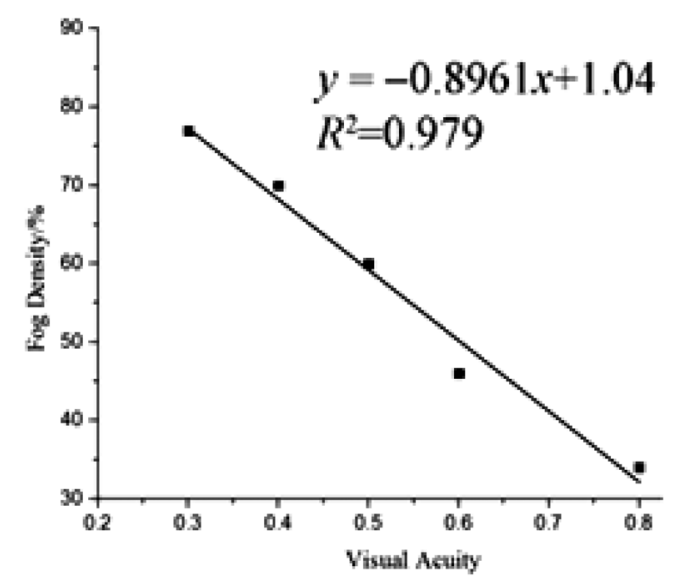

Figure 3 Relationship between visual acuity and average fog density. 
Table 2 Comparison of the results between different subject categories.

\begin{tabular}{|c|c|c|c|c|c|c|}
\hline Subject's visual acuity & & 0.3 & 0.4 & 0.5 & 0.6 & 0.8 \\
\hline \multirow[t]{2}{*}{ The average value of fog density without astigmatism } & Initial visual acuity below 1 & $77 \%$ & $71 \%$ & $64 \%$ & $52 \%$ & $35 \%$ \\
\hline & Initial visual acuity above 1 & $83 \%$ & $80 \%$ & $75 \%$ & $61 \%$ & $52 \%$ \\
\hline \multirow[t]{2}{*}{ The average value of fog density with astigmatism } & Initial visual acuity below 1 & $72 \%$ & $62 \%$ & $44 \%$ & $29 \%$ & $18 \%$ \\
\hline & Initial visual acuity above 1 & $77 \%$ & $73 \%$ & $62 \%$ & $50 \%$ & $37 \%$ \\
\hline
\end{tabular}

may be negatively correlated with fog density. Thus, a graph of the relationship between visual acuity and average fog density is presented in Figure 3. The visual acuity seems to be linearly related to average fog density with a fitting equation given by $y=-0.8961 x+1.04$ and $R^{2}=0.979$ being close to 1 . Thus, the curve is quite reliable. The influence of fog upon human visual acuity can be studied by the inverse function, namely, $y=-1.116 x+1.16$. Its slope reflects the speed of change of visual acuity with fog density, and the absolution of the slope can show the sensitivity of human vision in a fog environment.

During the experiment, we noticed that some subjects have astigmatism, which may cause individual differences. Initial visual acuity may likewise lead to such differences. Initial visual acuity can be classified into two categories: above 1.0 and below 1.0. Table 2 shows the calculated fog density for each subject category.

From the table above, it can be observed that subjects without astigmatism stay in higher fog density environments for a longer time compared with subjects with astigmatism of comparable initial visual acuity. Moreover, subjects with initial visual acuity above 1.0 stay in higher fog density environments for a longer time compared to those with initial visual acuity below 1.0. That is to say, subjects whose visual acuity is higher in normal situations also have better vision in fog environments. Concerning the speed of change of visual acuity, it can be seen that subjects without astigmatism or with initial visual acuity above 1.0 have higher sensitivity, indicating that their visual acuity decreases significantly as the density of fog increases.

\section{Conclusion}

In this study, human vision under different fog densities was investigated. Ultrasonic humidifiers were used to produce fog in a dark box, simulating a fog environment. In the process of fog dissipation, when achieving a specified level of vision, the corresponding fog density was calculated. The results are given below.

1. Visual acuity decreases linearly with fog density.

2. Astigmatism may cause differences between the subject's observations when using the Landolt C chart as a target. Subjects without astigmatism per- form better compared to subjects with astigmatism in the fog environment.

3. Initial visual acuity influences human eyesight in a fog environment. Subjects with better initial visual acuity can more easily reach a certain level of vision in fog environments.

\section{References}

(1) Shuai, H., Yu, T., Kun, L., Renxian, C., Xiaoli, Z., Xihong, B. and Muqing, L.: Study the transmittance properties of light sources under simulated hazy condition, China Illuminating Engineering Journal, 5, pp. 111-115 (2014).

(2) Kurniawan, B. A., Nakashima, Y., Takamatsu, M. and Kidoh, Y:: Visual perception of color LED light in dense fog, J. Light Visual Environ., 31-3, pp. 152-154 (2007).

(3) Zaini, M. F., Kurniawan, B. A., Nakashima, Y., et al.: Perceived brightness and saturation of color LED light in dense fog at night time, J. Light Visual Environ., 33-2, pp. 107-109 (2009).

(4) Babaria, B. R., Alvarez, T. L., Bergen, M. T., et al.: Transmission of light in a synthetic fog medium, bioengineering conference (2004), Proc. IEEE 30th Annual Northeast, IEEE, pp. 23-24 (2004).

(5) Mukaigawa, Y., Yagi, Y. and Raskar, R.: Analysis of Light Transport in scattering media, Computer Vision and Pattern Recognition (CVPR), 2010 IEEE Conference on, IEEE, pp. 153-160 (2010).

(6) Sirohi, R. S.: Effect of fog on the color of a distant light source, J. Phys. D Appl. Phys, 3-1, pp. 96-99 (1970).

(7) Li, C. and Peng, P.: Visibility measurement using multi-angle forward scattering by liquid droplets, Meas. Sci. Technol., 23-10, p. 105802 (2012).

(8) Xuefeng, G.: The Research for Visibility and Penetration of Different Electric Light Sources in Different Medium Density, ShenZhen University, 10590 (2011).

Part of this work is published at the 15th International Symposium on the Science and Technology of Lighting, May 2016, Kyoto, Japan. 